\title{
CRECIMIENTO COMPENSATORIO DE ALPACAS: EFECTO DE DIFERENTES NIVELES DE RESTRICCIÓN ENERGÉTICA
}

\author{
Compensatory Growth in Alpacas: Effect of Different Energy \\ Restriction LeVels
}

Juan Olazábal L. ${ }^{1,2}$, Felipe San Martín H. ${ }^{3}$, Miguel Ara G. ${ }^{3}$ y Francisco Franco F. ${ }^{1}$

\section{Resumen}

\begin{abstract}
El presente trabajo se realizó con el objetivo de evaluar y cuantificar el crecimiento compensatorio $(\mathrm{CC})$ en alpacas como respuesta a la restricción energética, medido en términos de ganancia de peso, consumo y conversión de alimento, e índice de recuperación. Se utilizaron 20 alpacas hembras destetadas que fueron sometidas a una fase de restricción energética y otra de compensación alimenticia de 60 días cada una. Los tratamientos durante la fase de restricción fueron: Sin restricción (SR): 3.5\% de materia seca (MS) del peso vivo (PV), Restricción moderada (Rmod): 2.5\% de MS del PV, Restricción media (Rme): $1.8 \%$ de MS del PV, y Restricción alta (Ral): $1.08 \%$ de MS del PV. Los animales recibieron el mismo alimento en forma ad libitum durante la fase de compensación. La tasa de crecimiento durante la fase de compensación varió de acuerdo al grado de restricción previa, siendo superior, en los animales con Rmod, seguido por Rme y Ral. Durante el CC, las alpacas con restricción alimenticia mostraron un mayor consumo y mejor conversión alimenticia; asimismo, las mayores tasas de crecimiento, mejor conversión e índice de recuperación se observaron en los periodos iniciales de la fase de compensación.
\end{abstract}

Palabras clave: crecimiento compensatorio, alpaca, consumo, conversión alimenticia

\section{AbSTRACT}

The present study was carried out to evaluate the compensatory growth (CG) in alpacas as the effect of a energy restriction period, measured in terms of body weight gain, feed intake, feed conversion and recover index. Twenty weaned female alpacas underwent a restricted feed intake phase and a compensatory phase of 60 days each. Treatments during the restriction phase were: No restriction level (NR): 3.5\% dry matter (DM) of body weight (BW), Moderate restriction level (Rmod): $2.5 \%$ DM of BW, Medium restriction level (Rme): $1.8 \%$ DM of BW, High restriction level (RH): $1.08 \%$ DM of BW. The animals were feed ad libitum with the same type of feed during the compensatory

\footnotetext{
${ }^{1}$ Estación Experimental del Centro de Investigación IVITA-Marangani, ${ }^{3}$ Laboratorio de Bioquímica, Nutrición y Alimentación Animal, Facultad de Medicina Veterinaria, Universidad Nacional Mayor de San Marcos, Lima

${ }^{2}$ E-mail: jolazaball@unmsm.edu.pe
} 
phase. The growth rate during the compensatory phase varied according to the level of feed restriction, being higher in animals under Rmod, followed by Rme and RH. During the CG period, alpacas with feed restriction showed higher feed intake and better feed conversion; furthermore, the higher growth rates, better conversion rates and recover index were observed during the first period of the compensatory phase.

Key words: compensatory growth, alpaca, intake, feed conversion

\section{INTRODUCCIÓN}

El crecimiento compensatorio (CC) es un proceso fisiológico por el cual un organismo acelera su tasa de crecimiento después de un periodo de desarrollo restringido, usualmente debido a la reducción del consumo de alimento (Owens et al., 1993; Hornick et al., 2000).

La alimentación de los camélidos sudamericanos en el altiplano peruano es exclusivamente al pastoreo sobre pasturas naturales (San Martín, 1996). La biomasa disponible en la región está influenciada por la marcada estacionalidad de la precipitación: una estación de lluvia (noviembre-abril) y otra de seca (mayo-noviembre). El crecimiento de las pasturas naturales durante la estación seca es reducido, lo que origina periodos donde no se cubren los requerimientos nutricionales de los animales. Uno de los periodos críticos de máxima restricción alimenticia es el destete (setiembre - octubre). Esto origina que el desarrollo corporal de las alpacas recién destetadas se retrase, tanto por la escasa disponibilidad de forraje como por el corte del suministro de leche materna. Es así que su normal crecimiento se encuentra afectado hasta la época de lluvias, donde puede ocurrir el CC, debido a la mayor disponibilidad de forraje (San Martín, 1996).

En la zona, la reproducción de las hembras se inicia, como práctica de manejo, a los dos años de edad, pese a que al año de edad presentan una conducta sexual (FernándezBaca y Novoa, 1968), tasas de ovulación y fertilización (Fernández-Baca et al., 1970), así como subsiguientes tasas de parición, peso corporal y tasas de supervivencia de la cría (Novoa et al., 1972) similares a las observadas en hembras adultas. No obstante, estas respuestas solamente se logran cuando las hembras alcanzan el $60 \%$ del peso $(36 \mathrm{~kg})$ de una hembra adulta $(60 \mathrm{~kg})$ al año de edad, y esto puede ser logrado dentro de ciertas condiciones de manejo y alimentación por efecto del CC.

El presente estudio fue diseñado para evaluar y cuantificar la respuesta de las alpacas a la restricción alimenticia, a través del CC, medido en términos de ganancia de peso, consumo y conversión de alimento, e índice de recuperación.

\section{Materiales y Métodos}

El estudio se realizó en la Estación Experimental del Centro de Investigación IVITA-Maranganí, de la Facultad de Medicina Veterinaria de la Universidad Nacional Mayor de San Marcos. El instituto se localiza a 3727 msnm, en el distrito de Maranganí, departamento del Cusco.

Se utilizaron 20 alpacas hembras destetadas en setiembre 2007, seleccionadas de acuerdo a la fecha de nacimiento y peso corporal y la etapa experimental se inició en noviembre. Los animales permanecieron en pastura cultivada (rye grass italiano, rye grass inglés y trébol blanco) durante la etapa de acostumbramiento. Para el estudio, los animales fueron dosificados contra parásitos internos y externos, y alojados en corrales individuales de $2.0 \times 1.5 \mathrm{~m}$. 
El experimento constó de una fase de restricción de alimentos y otra de compensación de 60 días cada una. El alimento ofrecido consistió en afrecho de cebada (92.7\%), harina de pescado $(6.3 \%)$ y una mezcla de vitaminas y minerales (1.0\%), en base a materia seca (MS), el cual contenía $13 \%$ de proteína cruda y $2.29 \mathrm{Mcal} / \mathrm{kg}$ de energía metabolizable.

Los tratamientos evaluados durante la fase de restricción alimenticia fueron:

- $\quad$ Sin restricción (SR). Alimento ad libitum (MS equivalente al 3.5\% del peso vivo [PV]).

- Restricción moderada (Rmod). Alimento necesario para conseguir el $50 \%$ de la ganancia de peso esperada en SR (MS equivalente al $2.5 \%$ del PV).

- Restricción media (Rme). Alimento suficiente para mantener el PV (MS equivalente al 1.8\% del PV).

- Restricción alta (Ral). Alimento que permita pérdida de PV (MS equivalente al $1.08 \%$ del PV).

Todos los animales recibieron el alimento ad libitum durante la fase de compensación. Los animales se pesaron cada 15 días durante todo el periodo del experimento. La ganancia de peso diario fue el coeficiente de regresión obtenido entre el peso vivo $(\mathrm{Y})$ y días de pesado (X). La ganancia de peso fue estimada para los periodos 0-28, 28-60 y 0-60 días en cada una de las dos fases.

El consumo de alimento se determinó diariamente, a través de la diferencia entre el alimento ofrecido y el rechazado, para los periodos 0-28, 28-60 y 0-60 días. La conversión de alimento se determinó a través de la relación entre el consumo de alimento y la ganancia de peso para los periodos $0-28,28$ 60 y 0-60 días en la fase de compensación.

El índice de recuperación (IR) se calculó para los periodos 0-28, 28-60 y 0-60 días de la fase de compensación, usando la fórmula desarrollada por Wilson y Osbourn (1960):
$\mathrm{IR}=(\mathrm{PIc}-\mathrm{PIcc})-(\mathrm{PFc}-\mathrm{PFcc})(\mathrm{PIc}-\mathrm{PIcc})$

Donde PIc y PIcc son los pesos del grupo control y restringido al inicio del periodo de compensación, respectivamente, y PFc y PFcc son los pesos de estos grupos al final del periodo de compensación.

Se empleó un diseño irrestricto al azar, con cuatro tratamientos y cinco repeticiones por tratamiento. La ganancia diaria de peso, consumo y conversión de alimento se analizó mediante análisis de varianza, y las diferencias encontradas se analizaron mediante la prueba de Duncan. Para el índice de recuperación de cada tratamiento restringido se estimó la medida de tendencia central (promedio).

\section{Resultados y Discusión}

Los pesos promedios de cada tratamiento en las fases de restricción y compensación se muestran en el Cuadro 1 . El crecimiento durante la fase de restricción reflejó los tratamientos impuestos $(\mathrm{p}<0.05)$, cumpliéndose las ganancias (o pérdidas) de peso esperadas. Así, los animales SR ganaron $35 \%$ de peso durante esta fase, mientras que los animales Rmod y Rme ganaron 16 y $4 \%$, respectivamente, y los animales Ral perdieron $24.3 \%$ del peso inicial.

Los incrementos de peso durante el primer periodo (0-28 d) de la fase de compensación para los animales de los tratamientos SR, Rmod, Rme y Ral fueron de 6, 17, 17 y $21 \%$, respectivamente, en el segundo periodo (28-60 d) fueron de 7, 11, 13 y 14\%, y durante toda la fase de compensación (0-60 d) fueron de $14,31,32$ y $39 \%$, respectivamente.

La ganancia de peso diario durante la fase de compensación fue menor $(p<0.05)$ en los animales SR en comparación con los animales de los demás tratamientos, no encontrándose diferencias entre los demás tratamientos (Cuadro 2). Al comparar las ga- 
Cuadro 1. Peso inicial y final de alpacas hembras destetadas, sometidas a una fase de restricción energética y otra de compensación alimenticia, en la sierra sur del Perú

\begin{tabular}{|c|c|c|c|c|c|}
\hline & \multicolumn{4}{|c|}{ Tratamientos $^{1}$} & \multirow{2}{*}{$\mathrm{CME}^{2}$} \\
\hline & SR & Rmod & Rme & Ral & \\
\hline \multicolumn{6}{|c|}{ Fase de restricción (0-60 d) } \\
\hline Inicial (kg) & $21.9^{\mathrm{a}}$ & $22.5^{\mathrm{a}}$ & $22.7^{\mathrm{a}}$ & $24.3^{\mathrm{a}}$ & 07.30 \\
\hline Final $(\mathrm{kg})$ & $29.6^{\mathrm{a}}$ & $26.1^{\mathrm{ab}}$ & $23.6^{\mathrm{bc}}$ & $18.4^{\mathrm{c}}$ & 17.08 \\
\hline \multicolumn{6}{|c|}{ Fase de compensación $(60-120 \mathrm{~d})^{3}$} \\
\hline Inicial (kg) & $29.6^{\mathrm{a}}$ & $26.1^{\mathrm{ab}}$ & $23.6^{\mathrm{bc}}$ & $18.4^{\mathrm{c}}$ & 17.08 \\
\hline $28 \mathrm{~d}(\mathrm{~kg})$ & $31.5^{\mathrm{a}}$ & $30.6^{\mathrm{a}}$ & $27.5^{\mathrm{ab}}$ & $22.3^{\mathrm{b}}$ & 17.94 \\
\hline Final (kg) & $33.7^{\mathrm{a}}$ & $34.1^{\mathrm{a}}$ & $31.1^{\mathrm{ab}}$ & $25.2^{\mathrm{b}}$ & 22.13 \\
\hline
\end{tabular}

nancias de peso entre periodos se observó que fue mayor en el periodo de $0-28 \mathrm{~d}$ que el periodo $28-60 \mathrm{~d}(\mathrm{p}<0.05)$, disminuyendo en el periodo $28-60 \mathrm{~d}$ en 33, 21 y $37 \%$ para los tratamientos Rmod, Rme y Ral, respectivamente.

En el Cuadro 2 se puede observar que el mayor índice de recuperación ocurrió durante el primer periodo de compensación ( 0 $28 \mathrm{~d}, \mathrm{p}<0.05)$. Los animales sometidos a una alimentación energética restringida crecieron a un ritmo superior a los animales sin restricción alimenticia durante la fase de compensación, expresando el fenómeno de CC, tal como es reportado en otras especies (Hornick et al., 2000). La expresión del CC fue diferenciada de acuerdo al grado de restricción previa, donde solo los animales con Rmod mostraron una compensación completa (114\%), mientras los de Rme mostraron una compensación parcial (57\%) y los de Ral tuvieron una pobre compensación (24\%).

Los diferentes grados de compensación hallados coinciden con reportes previos (Hornick et al., 2000). Así, la recuperación completa observada en animales con Rmod ha sido reportada en vacunos y las recuperaciones parciales de los animales con Rme y Ral ha sida reportada en ovinos; sin embargo, la compensación parcial es poco frecuente, y está más relacionada a una restricción en una edad muy temprana (Ryan et al., 1993). Generalmente, la recuperación es mayor cuando la duración de la restricción es corta y no demasiado severa. Así también, algunas fallas en la recuperación se pueden deber a la severidad de la restricción y a la calidad de la dieta utilizada durante la fase de compensación (Ryan et al., 1993). Drouillard et al. (1991) indicaron que la severidad de la restricción de nutrientes es determinante para la parcial o total recuperación del animal, más allá del tiempo de duración de la compensación, siendo difícil de conseguir cuando el periodo de restricción es muy prolongado.

El consumo de alimento durante la fase de compensación fue mayor en los animales restringidos en comparación con los SR $(p<0.05)$; y dentro de estos, fue mayor en el 
Cuadro 2. Ganancia de peso $(\mathrm{g} / \mathrm{d})$, índice de recuperación $(\%)$, consumo de alimento $(\mathrm{kg}$ MS/ $100 \mathrm{~kg} \mathrm{PV),} \mathrm{y} \mathrm{conversión} \mathrm{de} \mathrm{alimento} \mathrm{en} \mathrm{alpacas} \mathrm{hembras} \mathrm{destetadas,}$ durante una fase compensatoria de 60 días luego de haber pasado por una fase de restricción energética de 60 días

\begin{tabular}{cccccc}
\hline & \multicolumn{5}{c}{ Tratamientos $^{1}$} \\
\cline { 2 - 4 } & SR & Rmod & Rme & Ral & \\
\hline Ganancia de peso (g/d) & & & & & \\
0-28 d & 66.6 & 158.6 & 139.0 & 141.0 & $126.3^{\mathrm{a}}$ \\
28-60 d & 67.1 & 106.4 & 109.5 & 89.8 & $93.2^{\mathrm{b}}$ \\
0-60 d & $66.8^{\mathrm{a}}$ & $132.5^{\mathrm{b}}$ & $124.3^{\mathrm{b}}$ & $115.4^{\mathrm{b}}$ & 109.7 \\
Índice de recuperación (\%) & & & & & \\
0-28 d & 100 & 74 & 33 & 18 & \\
28-60 d & 100 & 134 & 35 & 8 & \\
0-60 d & 100 & 111 & 57 & 24 & \\
Consumo de alimento & & & & & \\
(kg MS/ 100 kg PV) & & & & & \\
0-28 d & 2.27 & 2.40 & 2.50 & 3.00 & $2.54^{\mathrm{a}}$ \\
28-60 d & 2.77 & 2.97 & 3.13 & 3.53 & $3.08^{\mathrm{b}}$ \\
0-60 d & $2.52^{\mathrm{a}}$ & $2.68^{\mathrm{b}}$ & $2.81^{\mathrm{b}}$ & $3.27^{\mathrm{c}}$ & 2.82 \\
Conversión de alimento & & & & & \\
0-28 d & 11.60 & 4.66 & 4.90 & 5.09 & $6.56^{\mathrm{a}}$ \\
28-60 d & 18.40 & 8.91 & 9.14 & 9.92 & $11.59^{\mathrm{b}}$ \\
0-60 d & $15.00^{\mathrm{a}}$ & $6.79^{\mathrm{b}}$ & $7.02^{\mathrm{b}}$ & $7.51^{\mathrm{b}}$ & 9.08 \\
\hline
\end{tabular}

${ }^{1}$ SR: sin restricción; Rmod: restricción moderada; Rme: restricción media; Ral: restricción alta

abc Letras diferentes dentro de filas en tratamientos y dentro de totales por parámetro productivo indican diferencia estadística $(p<0.05)$

grupo de mayor restricción (Ral, $\mathrm{p}<0.05$ ). Asimismo, el consumo de alimento fue significativamente mayor $(\mathrm{p}<0.05)$ durante la segunda etapa $(28-60$ d) de la fase compensatoria. Estos resultados coinciden con los reportes en otras especies (Kamalzadeh et al., 1997), donde se considera que la mejora observada en las etapas iniciales se debe a la mejora de la eficiencia en la utilización del alimento (Cartens et al., 1991), y la mejora en las etapas posteriores se atribuye a la cantidad de alimento consumido (Ryan et al., 1993; Santra y Pathak, 1999).

El mayor consumo de alimento se explica por una reducción del tejido adiposo durante el periodo de restricción, lo que origina un aumento del espacio de la cavidad abdominal, con reducción de la presión sobre el tracto digestivo. Además, una vez que el hígado y el tracto digestivo se restablecen du- 
rante la fase de compensación, se incrementa la síntesis de proteína con aumento de la demanda de energía que origina, a su vez, una retroalimentación positiva y aumento del consumo de alimento.

La conversión alimenticia, durante la fase de compensación, fue más eficiente en los animales con restricción energética que en los animales SR $(\mathrm{p}<0.05)$, no existiendo diferencias entre estos grupos (Cuadro 2). La mejor eficiencia de conversión se logra en el primer periodo $(p<0.05)$, donde la conversión alimenticia fue $37,48,46$ y $41 \%$ superior con respecto al segundo periodo, para los tratamientos SR, Rmod, Rme y Ral, respectivamente.

La mejor eficiencia alimenticia obtenida durante el primer periodo de compensación, coincide con otros estudios que mencionan que durante ese periodo los animales son más eficientes en el uso del alimento debido a la disminución de los requerimientos de mantenimiento durante la fase de restricción, disminución que se prolonga a la fase de compensación permitiendo que más nutrientes del alimento consumido se destine al crecimiento y menos al mantenimiento. Otra razón que explica este comportamiento es la menor relación grasa-proteína de los tejidos depositados durante la compensación (Cartens et al., 1991).

\section{Conclusiones}

- El crecimiento compensatorio se presentó en las alpacas después de un periodo de restricción energética.

- La tasa de crecimiento durante el crecimiento compensatorio varió de acuerdo al grado de restricción energética previa.

- Las alpacas, luego del periodo de restricción energética presentan un mayor consumo de alimento y una conversión alimenticia más eficiente que los animales que no fueron sometidos a dicha restricción.

- Las mayores tasas de crecimiento, mejor conversión alimenticia e índice de recuperación se presentaron en los periodos iniciales de la fase de compensación alimenticia.

\section{Literatura Citada}

1. Cartens GE, Johnson DE, Ellenberger MA, Tatum JD. 1991. Physical and chemical components of the empty body during compensatory growth in beef steers. J Anim Sci 69: 3251-3264.

2. Drouillard JS, Klopfenstein TJ, Britton RA, Bauer ML, Gramlich SM, Wester TJ, Ferrell CL. 1991. Growth, body composition, and visceral organ mass and metabolism in lambs during and after metabolizable protein or net energy restrictions. J Anim Sci 69: 3357-3375.

3. Fernández-Baca S, Hansel W, Novoa C. 1970. Embryonic mortality in the alpaca. Biol Reprod 3: 243-251.

4. Fernández-Baca S, Novoa C. 1968. Conducta sexual de la alpaca en empadre a campo. En: II Reunión ALPA. Lima: Asociación Latinoamericana de Producción Animal.

5. Hornick JL, Van Eenaeme C, Gérard O, Dufrasne I, Istasse L. 2000. Mechanisms of reduced compensatory growth. Domest Anim Endocrinol 19: 121-132.

6. Kamalzadeh A, Van Bruchem J, Koops WJ, Tamminga S, Zuart D. 1997. Feed quality restriction and compensatory growth in growing sheep: feed intake, digestion, nitrogen balance and modeling changes in feed efficiency. Livest Prod Sci 52: 209-217.

7. Novoa C, Fernández-Baca S, Sumar J, Leyva V. 1972. Pubertad en la alpaca. Rev Inv Pec, IVITA 1(1): 29-35.

8. Owens F, Dubeski P, Hanson C. 1993. Factors that alter the growth and development of ruminants. J Anim Sci 71:3138-3150. 
9. Ryan WJ, Williams IH, Moir RJ. 1993. Compensatory growth in sheep and cattle. I. Growth pattern and feed intake. Aust J Agric Res 44: 1609-1621.

10. San Martín F. 1996. Nutrición en alpacas y llamas. Lima: Púb Cienc IVITA No 27.29 p.
11. Santra A, Pathak NN. 1999. Nutrient utilization and compensatory growth in crossbred (Bos indicus x Bos taurus) calves. J Anim Prod 12: 1285-1291.

12. Wilson PN, Osbourn DF. 1960. Compensatory growth after undernutrition in mammals and birds. Biol Rev 35: 324-363. 